\title{
Oropouche virus cases identified in Ecuador using an optimised rRT-PCR informed by metagenomic sequencing
}

Authors: Emma L. Wise, Sully Márquez, Jack Mellors, Verónica Paz, Barry Atkinson,

Bernardo Gutierrez, Sonia Zapata, Josefina Coloma, Oliver G. Pybus, Simon K. Jackson, Gabriel Trueba, Gyorgy Fejer, Christopher H. Logue ${ }^{1}$ and Steven T. Pullan ${ }^{1}$.

${ }^{1}$ These authors contributed equally to this article.

Affiliations:

Public Health England, Salisbury, UK (E.L. Wise, C.H. Logue, J. Mellors, S.T. Pullan,) University of Plymouth, Plymouth, UK (E.L. Wise, C.H. Logue, S.K Jackson, G. Fejer) Microbiology Institute, Universidad San Francisco de Quito USFQ, Quito, Ecuador (S. Márquez, S. Zapata, G. Trueba, C.H. Logue)

Hospital Delfina Torres de Concha, Esmeraldas, Ecuador (V. Paz)

The Pirbright Institute, Woking, UK (B. Atkinson)

University of Oxford, Oxford, UK (B. Gutierrez, O.G. Pybus)

School of Biological and Environmental Sciences, Universidad San Francisco de Quito USFQ, Quito, Ecuador (B. Gutierrez).

University of California Berkeley School of Public Health, Berkeley, California, USA (J. Coloma)

Address for correspondence: Miss Emma L Wise, Public Health England Porton Down, Manor Farm Road, Salisbury, Wiltshire, SP4 0JG, UK; email: emma.wise@phe.gov.uk; telephone: 01980612230. 


\begin{abstract}
Oropouche virus (OROV) is responsible for outbreaks of Oropouche fever in parts of South America. We recently identified and isolated OROV from a febrile Ecuadorian patient, however, a previously published rRT-PCR assay did not detect OROV in the patient sample. A primer mismatch to the Ecuadorian OROV lineage was identified from metagenomic sequencing data. We report the optimisation of an rRT-PCR assay for the Ecuadorian OROV lineage, which subsequently identified a further five cases in a cohort of 196 febrile patients. We isolated OROV via cell culture and developed an algorithmically-designed primer set for whole-genome amplification of the virus. Metagenomic sequencing of the patient samples provided OROV genome coverage ranging from 68 - 99\%. The additional cases formed a single phylogenetic cluster together with the initial case. OROV should be considered as a differential diagnosis for Ecuadorian patients with febrile illness to avoid mis-diagnosis with other circulating pathogens.
\end{abstract}




\section{Introduction}

Oropouche virus (OROV) is the causative agent of Oropouche fever, an arboviral illness that is usually self-limiting and mild but in rare cases infects the central nervous system and causes meningitis $(1,2)$. The virus belongs to the genus Orthobunyavirus, family Peribunyaviridae, and has a negative-sense, single-stranded RNA genome composed of three segments. The small $(\mathrm{S})$ segment $(0.95 \mathrm{~kb})$ encodes the nucleoprotein $(\mathrm{N})$ and non-structural protein (NSs), the medium (M) segment $(4.36 \mathrm{~kb})$ encodes a polyprotein consisting of two glycoproteins (Gn, Gc) and one non-structural protein (NSm), whilst the large (L) segment (6.85 kb) encodes the RNA dependent RNA polymerase (RdRP) (3).

OROV is one of the most clinically important orthobunyaviruses in the Americas, with over half a million cases and more than 30 major outbreaks reported since it was first isolated in Trinidad and Tobago in 1955 (4). These figures are likely to be underestimates, caused in part by underreporting due to the similar clinical presentation of other arboviral diseases that co-circulate in the same areas, including dengue virus (DENV), chikungunya virus (CHIKV), Mayaro virus (MAYV), yellow fever virus (YFV) and Zika virus (ZIKV) $(4,5)$. Cases of Oropouche fever have been reported in Brazil, Peru, Panama, and Trinidad and Tobago (5). We recently isolated OROV for the first time from a patient in north-western Ecuador (6), suggesting that OROV may be circulating undetected in this region. This led us to further investigate the presence of OROV in an additional 196 febrile patients from the same coastal area of Esmeraldas, Ecuador, sampled in 2016.

Reverse transcription polymerase chain reaction (RT-PCR) is typically used for the detection of viral RNA directly from clinical samples (7-10). The rate of detection of OROV RNA in patient plasma during the first five days of illness is reported as $93.3 \%$ using a onestep real-time RT-PCR (rRT-PCR) (7). A disadvantage of RT-PCR is that a conserved target genome sequence is required, making it difficult to detect viral strains that are genetically 
divergent, reassortant, novel, or of an unexpected virus species. Metagenomic sequencing (untargeted sequencing of all genetic material in a sample) is an attractive alternative to conventional molecular detection methods as it does not require prior knowledge of pathogen genome sequences. For example, an established OROV rRT-PCR (7) was unable to detect OROV in the plasma of an Ecuadorian febrile patient who tested positive using metagenomic sequencing and virus isolation (6). This highlights the potential for misdiagnosis when screening for pathogens that lack a large collection of reference sequences on which to base targeted amplification. Here we report (i) the development of a rRT-PCR with improved sensitivity, (ii) an algorithmically-designed (11) primer set for whole-genome amplification of OROV, and (iii) the prevalence of OROV in the 2016 Esmeraldas patient cohort.

\section{Materials and methods}

\section{Patient samples}

Plasma samples were obtained from patients at Delfina Torres de Concha Hospital, Esmeraldas province, Ecuador, in $2016(n=196)$ and 2017 ( $n=62)$, along with metadata: patient age, sex, patient location, number of days of fever, and other clinical signs and symptoms. Use of these samples in this study was approved by the bioethics committee of Universidad San Francisco de Quito, Ecuador.

\section{RNA extraction and PCR assays}

Plasma samples were spiked with bacteriophage MS2 $\left(1.45 \times 10^{5} \mathrm{pfu}\right)$ as an internal control. RNA was extracted using the QIAamp Viral RNA mini kit (Qiagen) following manufacturer's instructions, substituting the addition of carrier RNA with the same volume of linear polyacrylamide (LPA). A negative extract control (molecular-grade water) was included in each batch of extractions. RNA was screened for DENV, CHIKV, ZIKV, YFV, 
MAYV, Plasmodium spp., Leptospira spp. and Rickettsia spp., using Public Health England in-house diagnostic PCR assays.

\section{Metagenomic sequencing}

DNAse treatment, cDNA preparation, random amplification by SISPA (Sequenceindependent Single Primer Amplification), and Illumina sequencing were performed as described previously (12). Reads were trimmed to remove adaptors and low-quality bases using trimmomatic (0.3.0) (13) with default parameters, to achieve an average phred score of Q30 across the read.

Taxonomic analysis of reads was undertaken using Centrifuge (Galaxy Version 1.0.3beta) (14) with index "Bacteria, Archaea, Viruses, Human", last updated on 12/06/2016, available at https://ccb.jhu.edu/software/centrifuge/manual.shtml, on a local instance of Galaxy (15). Mapping to OROV genomes was performed using BWA MEM (v0.7.15) (16), using OROV/EC/Esmeraldas/087/2016 (GenBank MF926352.1, MF926353.1, MF926354.1) as references. Quasibam (17) was used to generate consensus sequences, with a 5x coverage cut off. Mapping statistics were generated using SAMtools (v1.4) (18). Alignment and analysis of nucleotide and protein sequences was performed using the Clustal W method in MegAlign (v14.0.0). Human reads were removed by mapping to the human genome (human_g1k_v37[1000 genomes]), non-mapped reads were selected using SAMtools. De novo assembly of non-human reads was performed using SPAdes (v3.8.2) with --meta flag (19). A BLASTn (20) search was performed on resulting scaffolds larger than $1000 \mathrm{bp}$, against GenBank (nr/nt) database. Non-human reads were mapped (BWA MEM) to a multifasta file containing sequences from relevant viruses, including at least one representative genome from each PCR screening target (CHIKV, OROV, DENV1, DENV2, DENV3, DENV4, MAYV, YFV, ZIKV), any virus that had appeared during BLAST analysis 
of de novo assembled scaffolds (Hepatovirus A, Rift Valley Fever virus, Influenza A virus), and the internal control MS2.

Samples with $>0.02 \%$ reads assigned to a virus species by Centrifuge or an assembled scaffold larger than 1500 bp showing homology to a viral sequence using BLASTx were investigated for the presence of that specific virus. A positive virus detection was defined as $>40 \%$ genome coverage $(1 \mathrm{x})$ generated when mapping to a reference genome.

\section{Virus isolation}

Vero cells, freely provided by the European Collection of Authenticated Cell Cultures (ECACC; catalogue number 84113001), were inoculated with patient plasma diluted 1:10 in Eagle's minimum essential medium (MEM) to a volume of $1 \mathrm{~mL}$, incubated at $37^{\circ} \mathrm{C}$ with $5 \%$ $\mathrm{CO}_{2}$ for 96 hours. Cultures were sampled at 24-hour intervals, replication was determined using OROV rRT-PCR. Virus was harvested, filtered ( $0.2 \mu \mathrm{m}$ pore, Sartorius $)$ and stored at $80^{\circ} \mathrm{C}$.

\section{Primer alignment}

Primer and probe sequences were aligned using the ClustalW algorithm in the MEGA7 software package to a set of OROV N gene coding sequences (Genbank), representing the geographic (Brazil, Panama, Peru and Trinidad and Tobago) and temporal diversity of OROV isolates from the 1950s to present day (Gutierrez et al., preprint link (bioRxiv) pending).

rRT-PCR limit of detection analysis

For OROV rRT-PCR assay optimisation (supplementary material), a range of primers were tested (supplementary material, table S1) using Invitrogen Superscript III Platinum One- 
step Quantitative RT-PCR kit (Invitrogen) in a standard $25 \mu \mathrm{L}$ reaction, run on an ABI 7500 real-time PCR machine (Applied Biosystems). Optimal conditions were $18 \mu \mathrm{M}$ of each primer, $12.5 \mu \mathrm{M}$ probe and standard $\mathrm{MgSO}_{4}$ concentration. Cycling conditions: $50^{\circ} \mathrm{C}$ for 10 minutes, $95^{\circ} \mathrm{C}$ for 2 minutes, 45 cycles of $95^{\circ} \mathrm{C}$ for 10 seconds then $60^{\circ} \mathrm{C}$ for 40 seconds (with quantification analysis of fluorescence performed at the end of each $60^{\circ} \mathrm{C}$ step) and a final cooling step of $40^{\circ} \mathrm{C}$ for 30 seconds.

Absolute quantitation of RNA copy number was calculated from a standard curve of target sequence RNA, transcribed using the Megascript kit (Ambion). A 711 bp region of the S segment was amplified from cDNA of OROV/EC/Esmeraldas/087/2016, using primers incorporating a T7 promoter sequence (forward primer sequence GTC AGA GTT CAT TTT CAA CGA TGT ACC ACA ACG G, reverse primer sequence GAA ATT AAT ACG ACT CAC TAT AGG G CTC CAC TAT ATG TC). RNA was quantified using the Qubit RNA broad spectrum kit and purity confirmed using the RNA 6000 pico kit on a bioanalyzer (Agilent).

\section{Multiplex tiling PCR primer design}

The S, M and L segment sequences of OROV/EC/Esmeraldas/087/2016 were concatenated and inputted using default settings to the Primal Scheme (11) webtool (http://primal.zibraproject.org/), which implements an algorithm for designing multiplex PCR primers for virus lineages. The designed primer scheme was subsequently modified manually to account for segment ends, incorporating 5' and 3' end primers (see supplementary material for primer sequences).

RNA from patient sample D-057 and the cell-cultured prototype strain were amplified in two multiplex PCR reactions using the Ecuador OROV primers designed as described above. Primer pools were used at $100 \mu \mathrm{M}$. Products were visualised by gel electrophoresis 
and quantified using Qubit fluorometric quantitation. These were sequenced on a MinION (Oxford Nanopore, UK) and data processed as previously described (11).

\section{Results}

Development of an OROV rRT-PCR

An existing S segment assay (7) failed to detect OROV RNA in an Ecuadorian clinical sample that tested positive by both metagenomic sequencing and virus isolation (Figure 1) (6). The reverse primer (OROV R) (7) had two mismatches to the novel OROV/EC/Esmeraldas/087/2016 sequence. We attempted to improve sensitivity by analysing primer and probe suitability. Two new reverse primers were designed; one with mismatches corrected (Ec R) and one at a new site (Ec2 R) (supplementary material, table S1), based on an alignment of all publicly available OROV S segment sequences. Testing was performed against both OROV/EC/Esmeraldas/087/2016 and the 1955 prototype Trinidad and Tobago isolate from a distinct lineage (Genbank KP026181.1). The original reverse primer showed reduced sensitivity for the Ecuadorian strain, compared with the prototype strain (Figure 2). Primer Ec R showed improved sensitivity for the Ecuadorian strain, but reduced sensitivity for the prototype strain. Ec2 R improved sensitivity by three $\log$ f for the Ecuadorian strain and by one log for the prototype strain, compared with the original reverse primer. The final assay using Ec2 R underwent validation. The standard curve demonstrated a linear relationship between mean Cq value and log RNA copy number, the absolute limit of detection of the rRT-PCR assay was 10 copies of OROV RNA at a mean Cq value of 38.25 (95\% CI 37.8 to 38.7 ) (supplementary material, figure S1). No crossreactivity was observed in exclusivity testing against 23 virus species (supplementary material, table S2) within the Alphavirus, Flavivirus, Phlebovirus, Orthobunyavirus, Nairovirus and Mammarenavirus genera. Primer and probe sequences were aligned with all 
publicly available OROV N gene sequences to identify mismatches that may reduce sensitivity for certain strains. Of the 149 sequences, 19 had mismatch(es) to the forward primer, 21 sequences showed a mismatch to the probe, and 26 had mismatch(es) to the original reverse primer OROV R, 6 of which were mismatches at two positions (supplementary material, table S3). In contrast, no sequence has more than one mismatch to the new reverse primer Ec2 R; however, the number of sequences with a single mismatch was higher $(n=41)$.

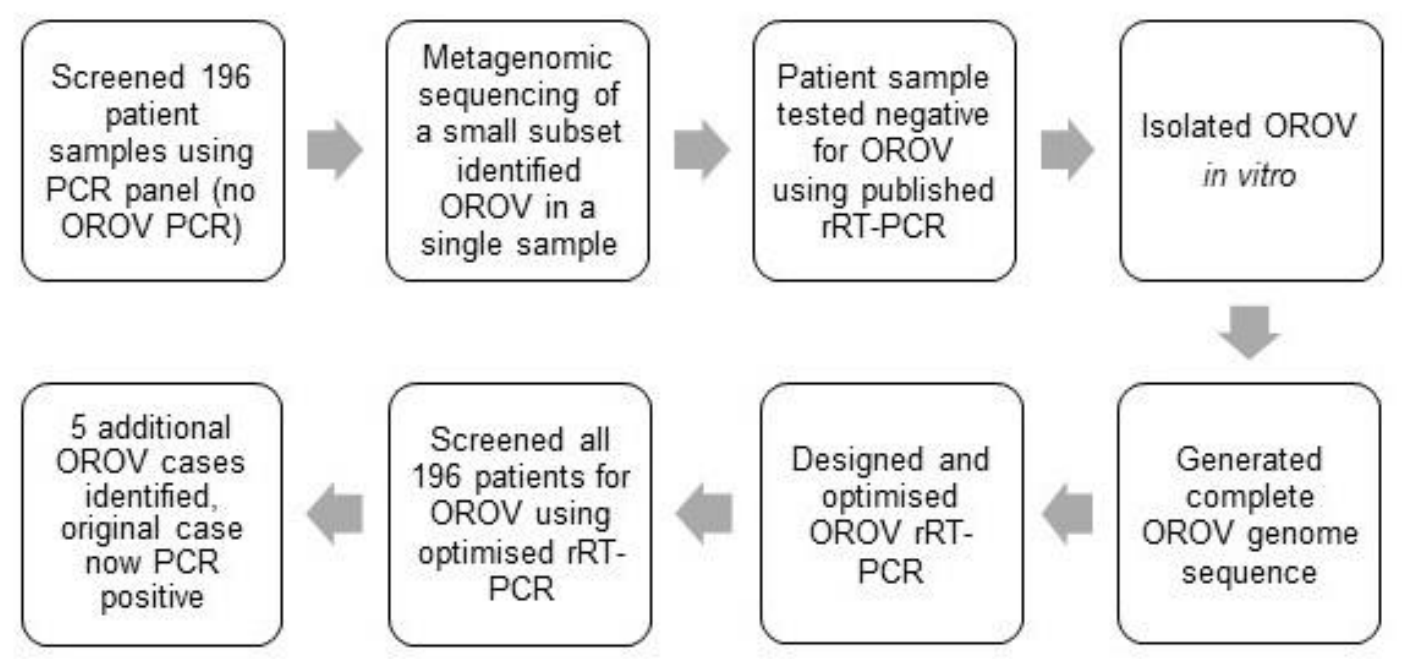

Figure 1. The workflow leading to the identification and isolation of OROV from 6 febrile Ecuadorian patients. 


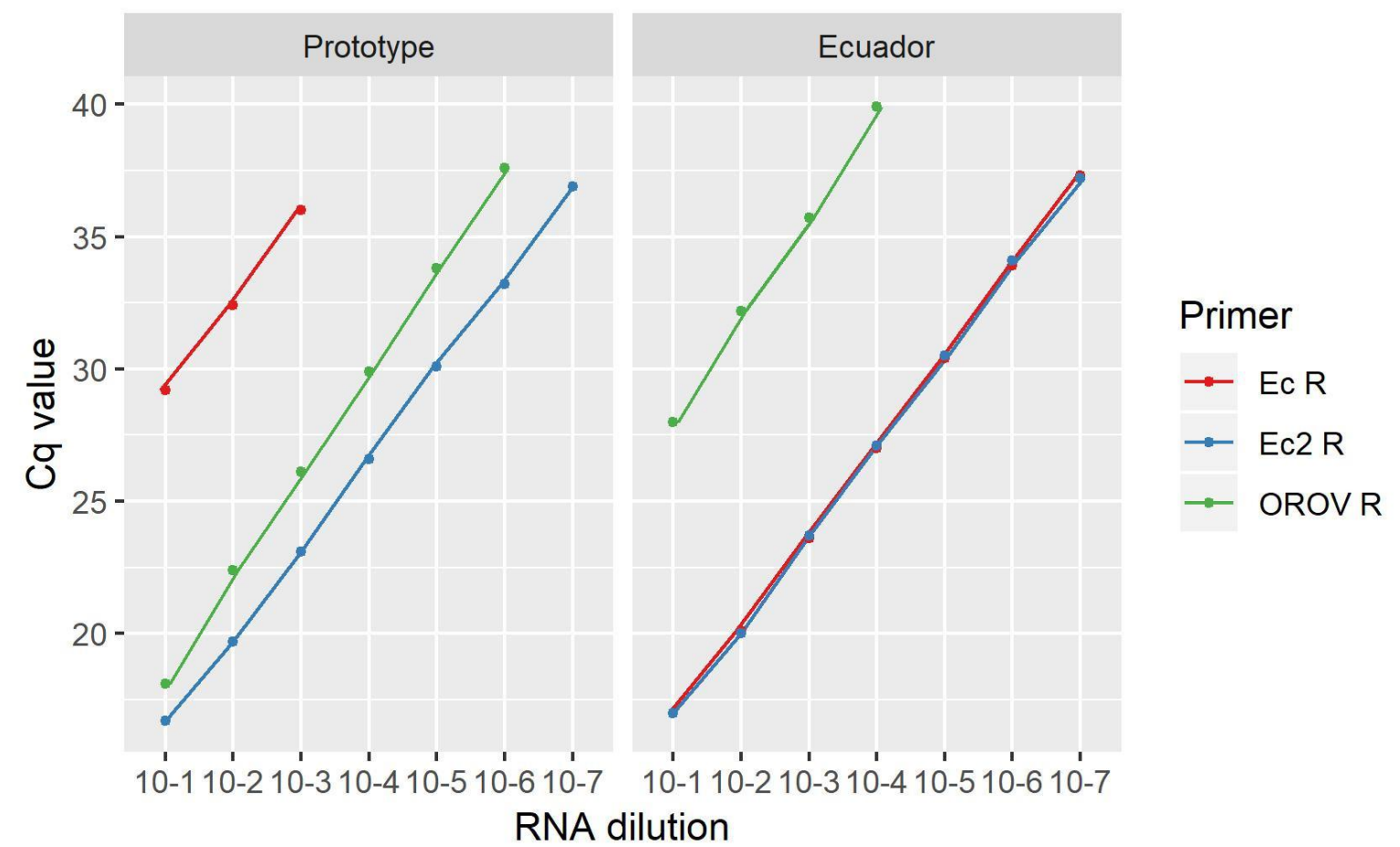

Figure 2. Sensitivity analysis of three reverse primers used in the rRT-PCR assay. Cq values are shown against a ten-fold serial dilution of OROV RNA, either from the prototype strain, or an Ecuadorian strain.

\section{Pathogen identification in febrile patients using PCR}

We initially used nine PCR assays to screen serum from Ecuadorian patients with undifferentiated febrile illness for pathogens previously reported from the country. Following our previous metagenomic sequencing based identification of OROV in one of these samples (6), and improvement of the OROV rRT-PCR, we re-screened all patient samples for OROV (Figure 1). PCR screening identified positives cases (Cq value <40) of ZIKV (prevalence 18.9\%,n=37), DENV (prevalence 3.6\%,n=7), OROV (prevalence 3.1\%,n=6), CHIKV (prevalence 1\%,n=2) and Leptospira spp. (prevalence 1\%, $n=2$ ) (Figure 3). No cases of MAYV, YFV, Plasmodium spp. or Rickettsia spp. were identified. 147 samples were 
negative for all pathogens tested. All samples were rRT-PCR-positive for internal control MS2.

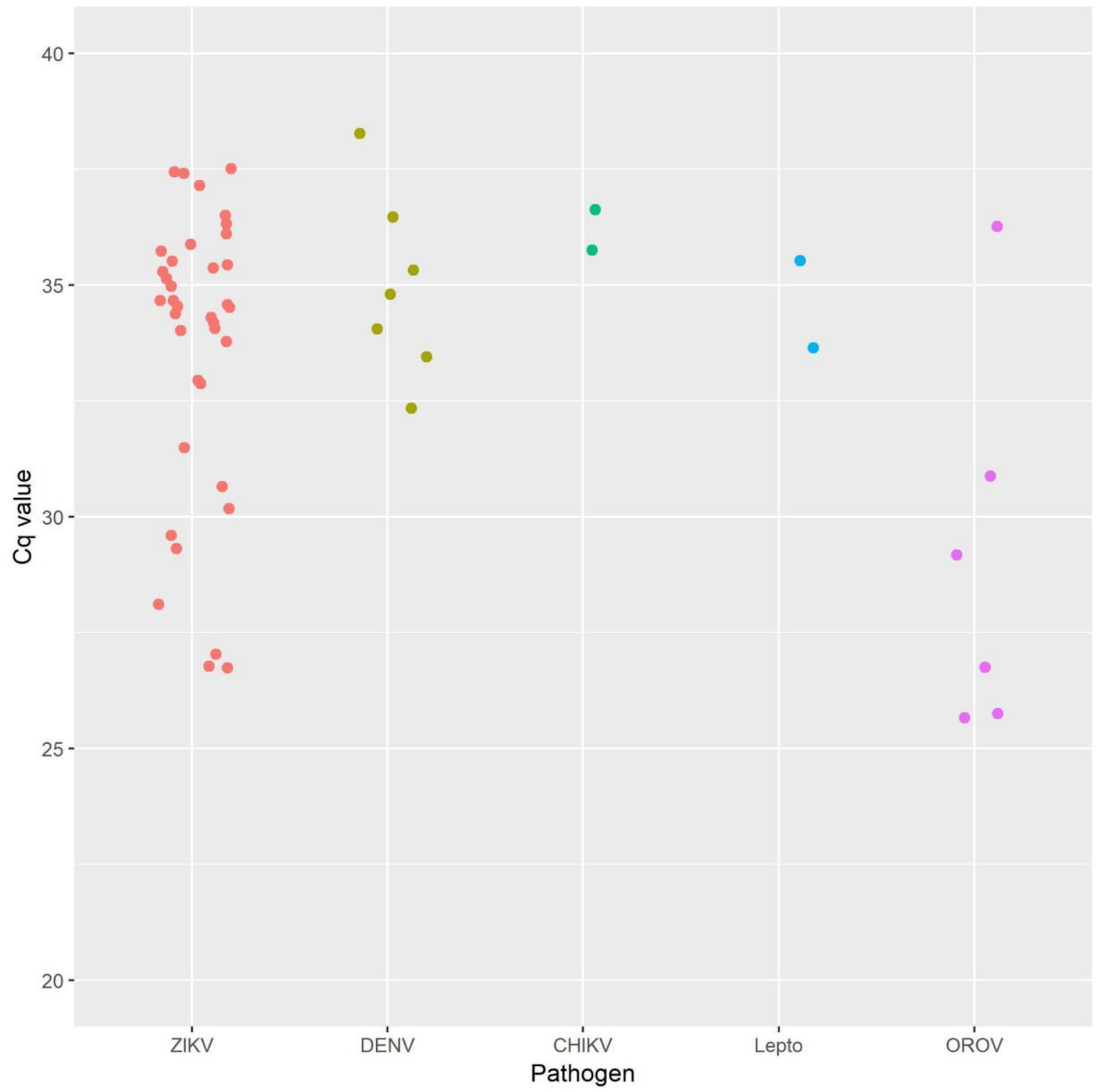

Figure 3. Cq values for pathogen positive plasma samples, screened by rRT-PCR. Lepto = Leptospira spp. 
Follow up screening of 2017 febrile patient samples

Sixty-two samples from febrile patients from Esmeraldas taken in 2017 were tested for OROV, CHIKV, DENV, MAYV, YFV, ZIKV and Rickettsia by PCR. All samples were negative for all pathogens and positive for internal control MS2.

Pathogen confirmation and genome characterisation using metagenomic sequencing

All OROV rRT-PCR-positive samples were also OROV positive by metagenomic sequencing, demonstrating that detection is possible from patient plasma samples with OROV RNA concentrations as low as $9.62 \times 10^{3}$ genome copies/mL (supplementary material, table S7). Mapping reads to reference sequence OROV/EC/Esmeraldas/087/2016 resulted in genome coverage ranging from 68 - 99\% (Figure 4). The proportion of OROVspecific reads does not appear to be particularly strongly correlated with $\mathrm{Cq}$ value, differences in the background level of non-viral RNA (in particular from the host) could account for this. It was still somewhat indicative of OROV genome coverage generated (Figure 4), with coverage generally decreasing as Cq increases. OROV reads were not detected in any of the 17 pathogen PCR-negative samples also tested. Hepatovirus A (HAV, formerly hepatitis A virus) was identified in one sample, with 1,894 reads providing $40 \%$ genome coverage (Table 1). Reads matching internal control MS2 were identified in all PCRnegative samples. 
A

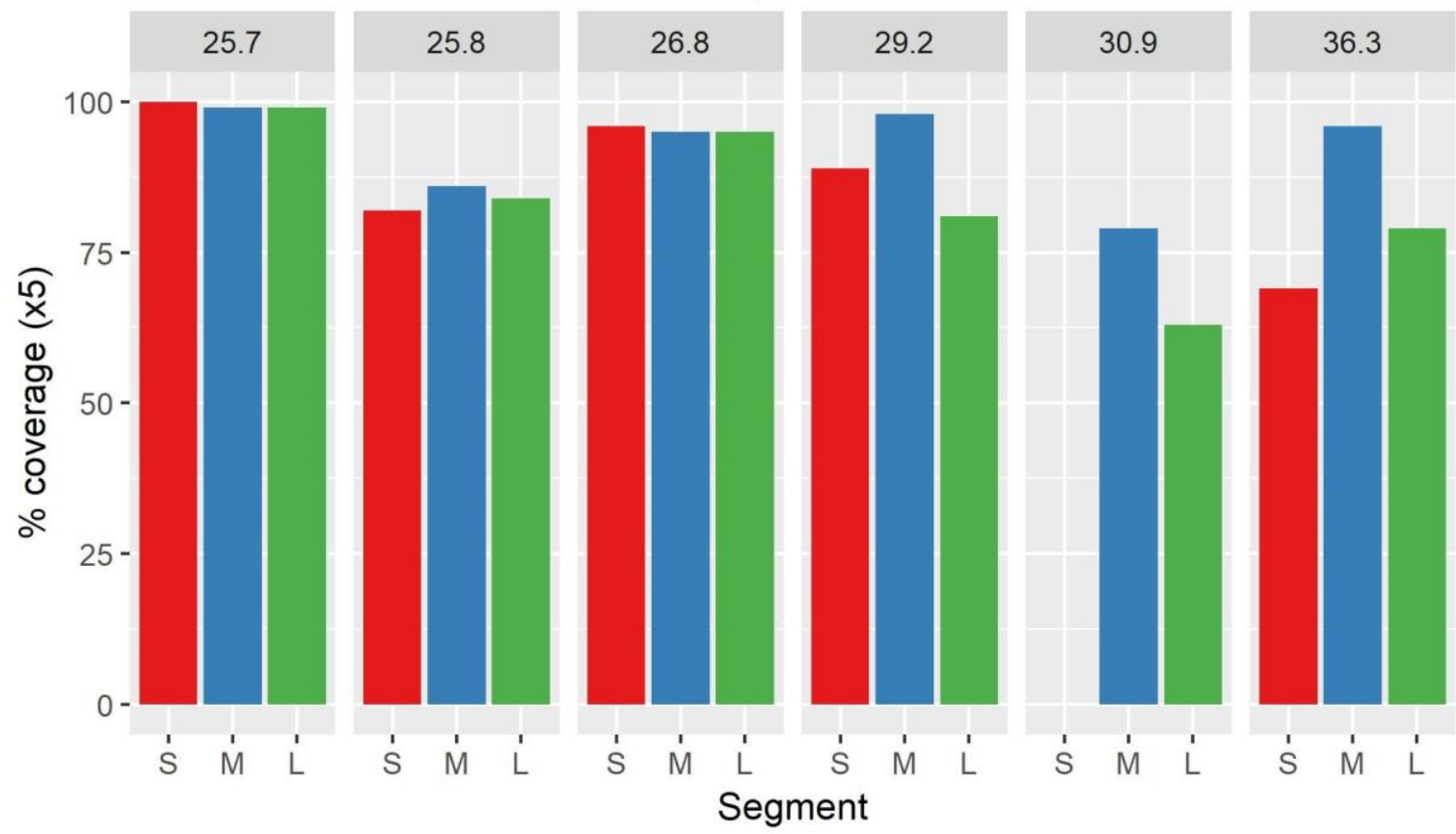

B

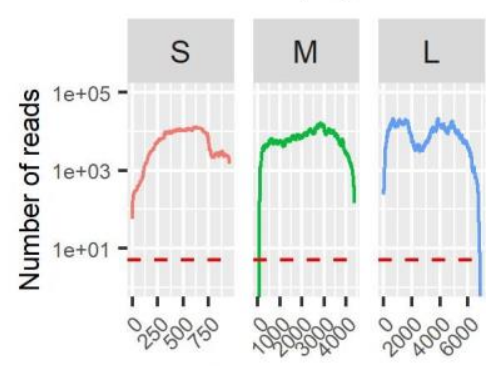

Patient D-210, Cq value 29.2

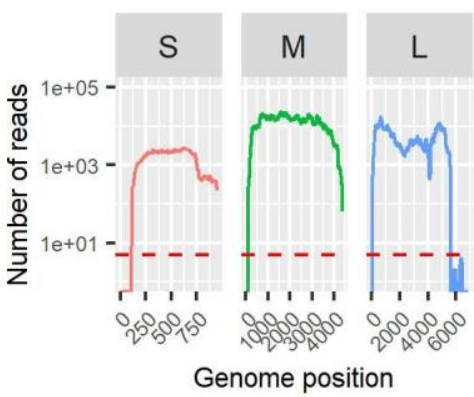

Patient D-155, Cq value 25.8

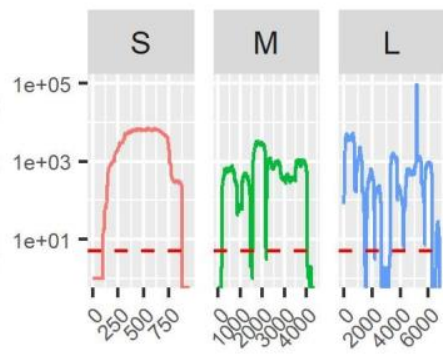

Patient D-206, Cq value 30.9

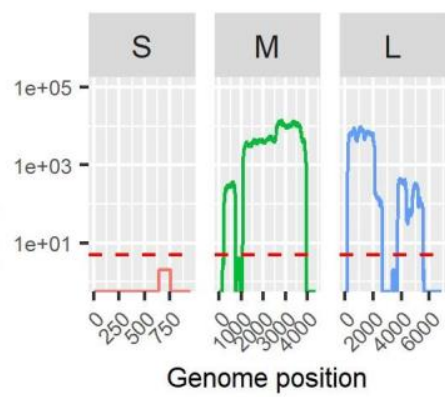

Patient D-171, Cq value 26.8

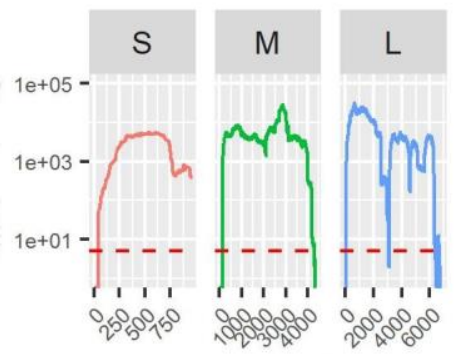

Patient D-087, Cq value 36.3

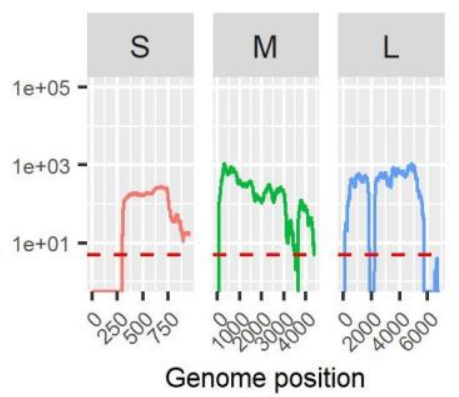

Figure 4. OROV genome coverage generated from metagenomic sequencing of OROV

positive patient plasma samples. Panel A: percentage genome coverage, separated by patient, rRT-PCR Cq value is indicated at the top of each plot. Coverage is defined as a minimum of 5 reads at any given position. For all data, mapping was to reference sequence 
OROV/EC/Esmeraldas/087/2016. Panel B: OROV genome coverage shown as number of reads at each genomic position. Plots are separated by genome segment (S, M, L). Red dashed line indicates $5 x$ coverage.

\begin{tabular}{|c|c|c|c|c|c|c|}
\hline $\begin{array}{c}\text { Sample } \\
\text { ID }\end{array}$ & $\begin{array}{c}\text { Virus } \\
\text { identified }\end{array}$ & $\begin{array}{l}\% \text { of total } \\
\text { reads } \\
\text { assigned by } \\
\text { Centrifuge }\end{array}$ & $\begin{array}{c}\text { Reads } \\
\text { assigned by } \\
\text { Centrifuge }\end{array}$ & $\begin{array}{c}\text { Reads } \\
\text { mapped to } \\
\text { reference } \\
\text { sequence }\end{array}$ & $\begin{array}{l}\text { 1x genome } \\
\text { coverage } \\
(\%)\end{array}$ & $\begin{array}{l}\text { Mapping } \\
\text { reference } \\
\text { (accession } \\
\text { number) }\end{array}$ \\
\hline D-005 & HAV & 0.02 & 478 & 1,894 & 40 & LC049339 \\
\hline D-057 & OROV & 7.42 & 143,925 & 870,960 & 99 & $\begin{array}{c}\text { MF926352.1 - } \\
54.1\end{array}$ \\
\hline D-087 & OROV & 0.70 & 5,084 & 9,972 & 80 & $\begin{array}{c}\text { MF926352.1 - } \\
54.1\end{array}$ \\
\hline D-155* & OROV & 1.97 & 66686 & 24,274 & 90 & $\begin{array}{c}\text { MF926352.1 - } \\
54.1\end{array}$ \\
\hline D-171* & OROV & 3.13 & 94,248 & 145,146 & 95 & $\begin{array}{c}\text { MF926352.1 - } \\
54.1\end{array}$ \\
\hline D-206 & OROV & 0.63 & 25,050 & 277,019 & 68 & $\begin{array}{c}\text { MF926352.1 - } \\
54.1\end{array}$ \\
\hline D-210 & OROV & 4.15 & 78,747 & 733,986 & 92 & $\begin{array}{c}\text { MF926352.1 - } \\
54.1\end{array}$ \\
\hline
\end{tabular}

Table 1. Viruses identified in patient samples by metagenomic sequencing. Cut-off value used was $\geq 40 \%$ genome coverage (from mapping reads to reference genome). *Data are from two separate sequencing runs combined.

Confirmation of OROV infection in PCR-positive patients, by virus isolation

OROV was successfully cultured in Vero cells from all five OROV positive patient plasma samples (virus was isolated from the initial patient D-087 as previously reported (6)). 
Genome copy numbers for all samples increased by at least $5 \operatorname{logs}$ to $1 \times 10^{12}$ copies $/ \mathrm{mL}$ at 72 hours post-infection (supplementary material, figure S2), demonstrating OROV was replicating. RNA from cultured viral supernatant (one passage in Vero cells) underwent metagenomic sequencing and the resulting reads were mapped to reference sequence OROV/EC/Esmeraldas/087/2016. Complete genomes were generated from all isolates and are available on Genbank, accession numbers MK506818 - MK506832.

The genomes were $99.7-100 \%$ identical to one another in the S segment and 99.9-100\% identical in the M and L segments. Single nucleotide polymorphisms (SNPs) were observed at 33 positions throughout the genome, all of which occurred in coding regions (supplementary material, table S4). Alignment of protein sequences showed that the $\mathrm{N}$ protein was $100 \%$ identical between isolates, whilst the NSs protein had a single amino acid substitution in isolate D-206. Isolate D-210 had two amino acid substitutions in the M protein, whilst three isolates (D-057, D-155 and D-206) had one substitution in this protein. Two isolates (D-155 and D-210) had a single amino acid substitution in the L protein (supplementary material, table S5). Of the eight substitutions seen, five constitute a reactive (R) group change and therefore may influence the structure or function of the protein.

The genome sequences from the patient and cultured sequences were compared to identify SNPs incurred during passage. The number of SNPs per genome ranged from one (sample D-057) to 15 (sample D-155) (supplementary material, table S6). The majority of changes were from a mixed population (defined as no one base occupying $>80 \%$ of reads) in the patient, to either a different mixed population, or a majority population (majority defined at a base occupying $>80 \%$ reads at a position) in the cultured isolate. Changes from a majority population in the patient were less common, seen at two (samples D-171 and D-210) to five (sample D-087) positions, with sample D-057 showing no changes of this type. None 
of the positions with polymorphisms between patient and cultured sequences were conserved between isolates.

\section{Development of an OROV multiplex tiling PCR primer scheme}

Samples with lower levels of viral RNA often present problems in generating complete genome sequences using the metagenomic sequencing approach (Table 1, Figure 4). To address this problem, a set of targeted genome amplification primers was designed using the algorithmic method described by Quick et al. (11). Using a cut-off of 20x read depth, $>99 \%$ coverage of all three Ecuadorian OROV genome segments was obtained (average coverage of 7,075x , 9,894x and 7,381x for the S, M and L segments, respectively) directly from clinical sample D-057 (Figure 5). For the prototype Trinidad and Tobago genome, $98 \%$ of the S segment (average coverage $=126,860 \mathrm{x}$ ), $93 \%$ of the $\mathrm{M}$ segment (average coverage $=119 x)$ and $92 \%$ of the $L$ segment (average coverage $=189 x)$ were obtained, despite its sequence divergence from the Ecuadorian strains.
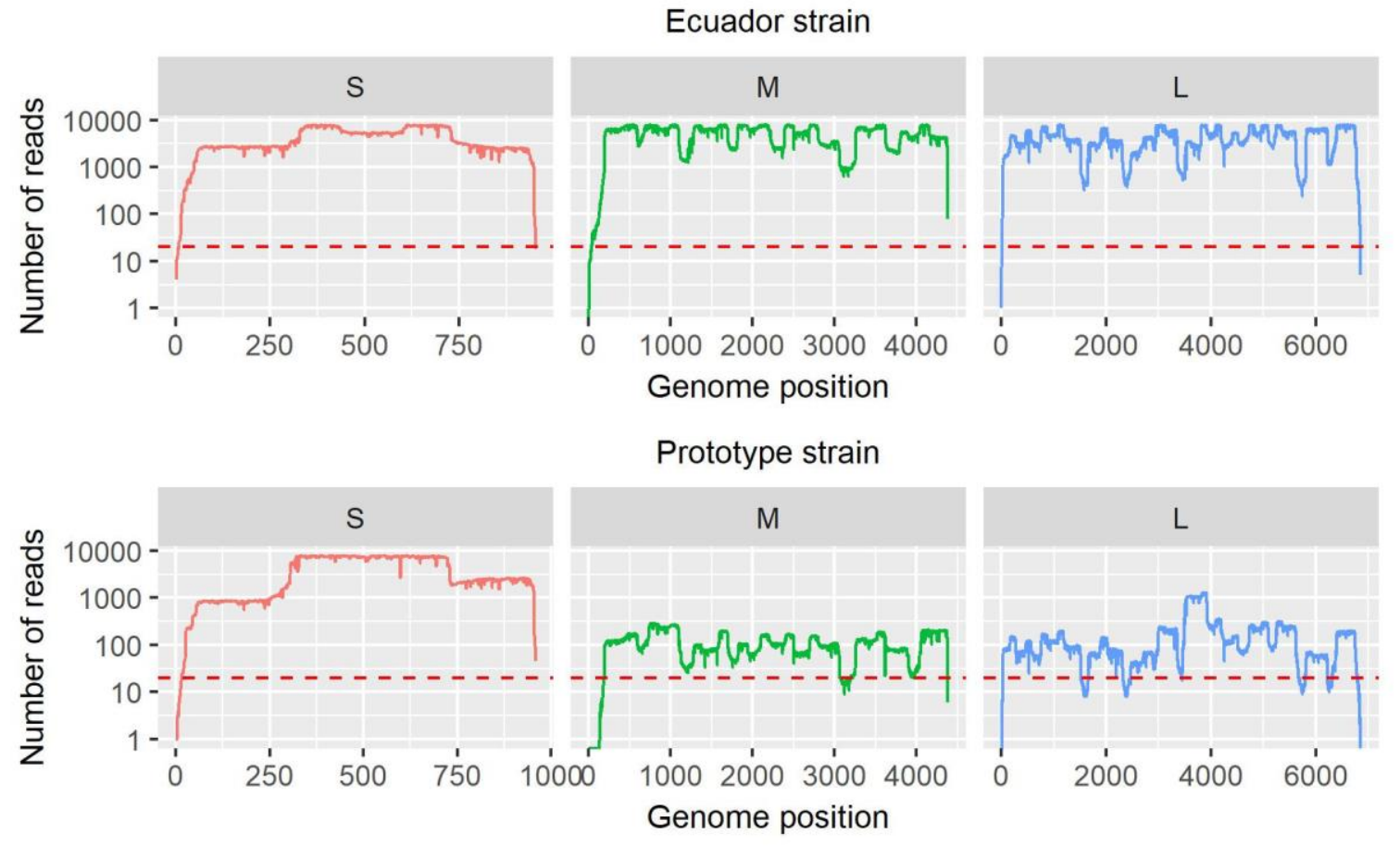
Figure 5. Genome coverage of two strains of OROV, sequenced on a MinION (Oxford Nanopore) from multiplex tiling PCR amplicons. Plots are separated by genome segment (S, M, L). Red dashed line indicates 20x coverage.

\section{Discussion}

Using metagenomic sequencing followed by screening using an optimised rRT-PCR, we have detected a cluster of six OROV cases from patients local to Esmeraldas, Ecuador, providing further evidence that OROV may be causing an unrecognised burden of human disease in previously unreported areas.

Some of these cases were not detected by the previously published assay due to a primer mismatch with the Ecuadorian genomes, which was identified in the metagenomic sequencing data. This discovery highlights the problems in developing amplification-based detection assays for pathogens with limited genomes, and the benefits offered by unbiased sequencing technologies. Although the cost associated with metagenomic sequencing may be prohibitive for frontline diagnosis in developing countries, it can be used in prospective screening studies to identify potential issues with rRT-PCR assays and allow for more alterations to increase inclusivity as reported here. We developed a new reverse primer for the previously-published assay that improves sensitivity for both the Ecuadorian strain (capable of detecting 10 copies of target RNA) and the genetically divergent prototype strain. Other OROV strains were not available to validate against, however an alignment of all publicly available strains showed that the majority of sequences are homologous to the primer/probe sequences. The rRT-PCR amplifies a region in the S segment, the target sequence of which is shared by a number of reassortant orthobunyaviruses, including Iquitos virus, which is known to circulate in neighbouring Peru and can cause Oropouche fever (21). This modified assay also has the potential to detect reassortant orthobunyaviruses containing 
OROV S segment. An algorithmically-designed multiplex primer set was developed that can amplify the majority of the genome from both Ecuadorian and prototype strains and may be a useful tool for sequencing OROV genomes from clinical samples with low viral titres.

Virus identification in the Ecuadorian cohort was undertaken initially using PCR. In addition to OROV, screening identified the presence of ZIKV, DENV, CHIKV and Leptospira spp. nucleotide sequences. These pathogens are known to circulate in Ecuador (22-24); ZIKV in particular was widespread in the Americas (including Ecuador) at the time of sampling (25), so the identification of a relatively large number of ZIKV cases $(n=37)$ was not unexpected. Cq values of 35-40 were observed for multiple samples across all identified pathogens; samples showing values in this range require confirmatory testing to be certain of the presence of the pathogen.

Metagenomic sequencing of 17 PCR-negative samples identified HAV sequence in one patient; no other viruses were conclusively identified. Ecuador is considered an 'intermediate endemicity' country for HAV (26) and therefore identification of this virus is not unexpected. The lack of viral sequences from the majority of the patient samples could be explained by a number of factors including clearance of virus from the blood by the time of sampling, or fever caused by non-infectious disease. Metagenomic sequencing successfully identified OROV in all OROV rRT-PCR-positive patients, with good genome coverage (68$99 \%$ ), suggesting that this method is effective for both detection and characterisation of OROV genomes from clinical samples.

The generation of complete viral genomes, using metagenomic sequencing or a multiplex tiling PCR (as opposed to the small fragments generated using conventional rRTPCR) enables more detailed and accurate molecular epidemiological analyses (27). Initial phylogenetic analysis of S, M, and L segments shows that the five additional Ecuadorian genomes cluster in a monophyletic group with our previously reported Ecuadorian strain, 
suggesting the possibility of local transmission within Esmeraldas. An investigation of potential OROV vector species in Esmeraldas would be highly informative. The group is most closely related to strain TVP-19256/IQE-7894 from Peru, 2008 (KP795086). A comprehensive phylogenetic analysis of OROV in South America, including the Ecuadorian genomes, has been undertaken (Gutierrez et al., preprint link (bioRxiv) pending).

It is well-documented that mutations occur during passaging in vitro (28). Our study generated complete genome sequences from viruses that had undergone one passage in Vero cells. The single passage was necessary to amplify virus and generate complete genome sequences, however, passaging was limited to minimise the number of mutations between patient and cultured viral genomes. A number of positions in the OROV patient genomes show a mixed population of variants, which progressed to a majority population in the cultured genome. Majority-majority changes were rare, and none of the SNPs observed between a patient and cultured genome were shared between strains.

No cases of OROV were detected during follow-up rRT-PCR screening of 62 patient samples from Esmeraldas, 2017. Previous outbreaks of Oropouche fever have been episodic and self-limited in nature (4); the six cases identified from 2016 could represent a single outbreak that ended that year. Alternatively, if OROV transmission did occur in Esmeraldas in 2017, it could have been missed due to the limited sample size. A larger-scale study is necessary to determine the prevalence and distribution of OROV in the Ecuadorian population.

OROV infection should be considered when diagnosing Ecuadorian patients with febrile illness. We hope that the molecular tools developed in this study will be useful for laboratories performing viral diagnostics and surveillance in the Americas. Very little is known about the dynamics of OROV in Ecuador or endemicity in neighbouring regions; the 
detection of OROV in multiple febrile patients, described here, warrants further investigation into its prevalence, associated vectors, and transmission.

\section{Acknowledgements}

This work was funded by Public Health England as part of a PHE-funded PhD studentship awarded to EW.

\section{Biographical sketch}

Miss Wise is a PhD student registered with Plymouth University and funded by Public Health England. Her research interests are emerging viral infections and viral immunology.

\section{References}

1. Pinheiro FP, Rocha AG, Freitas RB, Ohana BA, Travassos da Rosa AP, Rogério JS, et al. Meningitis associated with Oropouche virus infections. Rev Inst Med Trop Sao Paulo. $1982 \mathrm{Jul} ; 24(4): 246-51$.

2. Bastos M de S, Figueiredo LTM, Naveca FG, Monte RL, Lessa N, Pinto de Figueiredo $\mathrm{RM}$, et al. Identification of Oropouche Orthobunyavirus in the cerebrospinal fluid of three patients in the Amazonas, Brazil. Am J Trop Med Hyg. 2012 Apr;86(4):732-5.

3. Elliott RM. Orthobunyaviruses: recent genetic and structural insights. Nat Rev Microbiol. 2014 Oct;12(10):673-85.

4. Travassos da Rosa JF, de Souza WM, Pinheiro F de P, Figueiredo ML, Cardoso JF, Acrani GO, et al. Oropouche Virus: Clinical, epidemiological, and molecular aspects of a neglected orthobunyavirus. Am J Trop Med Hyg. 2017 May;96(5):1019-30. 
5. Sakkas H, Bozidis P, Franks A, Papadopoulou C. Oropouche Fever: A review. Viruses. 2018;10(4):175.

6. Wise EL, Pullan ST, Márquez S, Paz V, Mosquera JD, Zapata S, et al. Isolation of Oropouche virus from febrile patient, Ecuador. Emerg Infect Dis. 2018 May;24(5):935-7.

7. Weidmann M, Rudaz V, Nunes MRT, Vasconcelos PFC, Hufert FT. Rapid detection of human pathogenic orthobunyaviruses. J Clin Microbiol. 2003 Jul;41(7):3299-305.

8. Nunes MRT, de Souza WM, Savji N, Figueiredo ML, Cardoso JF, da Silva SP, et al. Oropouche orthobunyavirus: Genetic characterization of full-length genomes and development of molecular methods to discriminate natural reassortments. Infect Genet Evol. 2018 Nov 29;68:16-22.

9. Naveca FG, Nascimento VA do, Souza VC de, Nunes BTD, Rodrigues DSG, Vasconcelos PF da C. Multiplexed reverse transcription real-time polymerase chain reaction for simultaneous detection of Mayaro, Oropouche, and Oropouche-like viruses. Mem Inst Oswaldo Cruz. 2017 Jul;112(7):510-3.

10. Pauvolid-Corrêa A, Campos Z, Soares R, Nogueira RMR, Komar N. Neutralizing antibodies for orthobunyaviruses in Pantanal, Brazil. PLoS Negl Trop Dis. 2017 Nov;11(11):e0006014.

11. Quick J, Grubaugh ND, Pullan ST, Claro IM, Smith AD, Gangavarapu K, et al. Multiplex PCR method for MinION and Illumina sequencing of Zika and other virus genomes directly from clinical samples. Nat Protoc. 2017 Jun;12(6):1261-76.

12. Kafetzopoulou LE, Efthymiadis K, Lewandowski K, Crook A, Carter D, Osborne J, et al. Assessment of metagenomic Nanopore and Illumina sequencing for recovering whole genome sequences of chikungunya and dengue viruses directly from clinical samples. Eurosurveillance. 2018 Dec;23(50). 
13. Lohse M, Bolger AM, Nagel A, Fernie AR, Lunn JE, Stitt M, et al. RobiNA: a userfriendly, integrated software solution for RNA-Seq-based transcriptomics. Nucleic Acids Res. 2012;40(W1):W622-7.

14. Kim D, Song L, Breitwieser FP, Salzberg SL. Centrifuge: rapid and sensitive classification of metagenomic sequences. Genome Res.2016. Dec;26(12):1721-1729.

15. Goecks J, Nekrutenko A, Taylor J, Galaxy Team. Galaxy: a comprehensive approach for supporting accessible, reproducible, and transparent computational research in the life sciences. Genome Biol. 2010 Aug 25;11(8):R86.

16. Li H. Aligning sequence reads, clone sequences and assembly contigs with BWA-MEM [Internet]. 2013 [cited 2019 Jan 24]. Available from: http://arxiv.org/abs/1303.3997

17. Penedos AR, Myers R, Hadef B, Aladin F, Brown KE. Assessment of the utility of whole genome sequencing of measles virus in the characterisation of outbreaks. PLoS One. 2015 Nov 16;10(11):e0143081.

18. Li H, Handsaker B, Wysoker A, Fennell T, Ruan J, Homer N, et al. The sequence alignment/map format and SAMtools. Bioinformatics. 2009 Aug 15;25(16):2078-9.

19. Bankevich A, Nurk S, Antipov D, Gurevich AA, Dvorkin M, Kulikov AS, et al. SPAdes: a new genome assembly algorithm and its applications to single-cell sequencing. J Comput Biol. 2012 May;19(5):455-77.

20. Altschul S. Basic Local Alignment Search Tool. J Mol Biol. 1990;215(3):403-10.

21. Aguilar PV, Barrett AD, Saeed MF, Watts DM, Russell K, Guevara C, et al. Iquitos Virus: A novel reassortant orthobunyavirus associated with human illness in Peru. PLoS Negl Trop Dis. 2011;5(9):e1315.

22. Barragan V, Chiriboga J, Miller E, Olivas S, Birdsell D, Hepp C, et al. High leptospira diversity in animals and humans complicates the search for common reservoirs of human disease in rural Ecuador. PLoS Negl Trop Dis. 2016 Sep;10(9):e0004990. 
23. Cifuentes SG, Trostle J, Trueba G, Milbrath M, Baldeón ME, Coloma J, et al. Transition in the cause of fever from malaria to dengue, Northwestern Ecuador, 1990-2011. Emerg Infect Dis. 2013 Oct;19(10):1642-5.

24. Zambrano H, Waggoner JJ, Almeida C, Rivera L, Benjamin JQ, Pinsky BA. Zika virus and chikungunya virus coinfections: A series of three cases from a single center in Ecuador. Am J Trop Med Hyg. 2016 Oct 5;95(4):894-6.

25. Ministry of Public Health of Ecuador 2016. Casos confirmados autóctonos e importados de ZIKV. Ecuador Hasta la SE 47 [Internet]. [cited 2019 Feb 19]. Available from: http://www.salud.gob.ec/wp-content/uploads/2015/12/GACETA-ZIKA-SEM47.pdf

26. Jacobsen KH, Wiersma ST. Hepatitis A virus seroprevalence by age and world region, 1990 and 2005. Vaccine. 2010;28(41):6653-7.

27. Dudas G, Bedford T. The ability of single genes vs full genomes to resolve time and space in outbreak analysis [Internet]. bioRxiv. 2019 [cited 2019 Apr 18]. p. 582957. Available from: https://www.biorxiv.org/content/10.1101/582957v1.abstract

28. Domingo E, Holland JJ. RNA virus mutations and fitness for survival. Vol. 51, Annu Rev Microbiol. 1997;51:151-78. 